\title{
A PROPERTY OF ARITHMETIC SETS
}

\author{
HISAO TANAKA
}

\begin{abstract}
We shall show that every nonempty countable arithmetic subset of $N^{N}$ contains at least one element $\alpha$ such that the singleton $\{\alpha\}$ itself is arithmetic. The proof is carried out by using a method in classical descriptive set theory.
\end{abstract}

It is known that $(*)$ if no member of a nonempty $\Sigma_{1}^{1}$ set $E$ is hyperarithmetic then $E$ contains a perfect subset. (In this note, sets mean subsets of $N^{N}$-the set of all 1-place number-theoretic functions which we identify with Baire zero-space.) In fact, every $\Sigma_{1}^{1}$ set with a nonhyperarithmetic element contains a perfect subset. (See, e.g., Harrison [2, Theorem 2.12] and Mathias [4, T3200].) In what follows, we shall show that an arithmetic counterpart of the proposition $(*)$ holds true:

THEOREM 1. If no member of a nonempty arithmetic set $A$ is an arithmetic singleton, then A contains a perfect subset.

It is evident that one can not replace "arithmetic singleton" by "arithmetic element" in our theorem.

T. G. McLaughlin has asked the following question (unpublished): Let $A$ be a nonempty countable arithmetic set. Then, must some member of $A$ be an arithmetic singleton? Now we can obtain an affirmative answer to this question as a direct corollary of our theorem, thus:

Corollary 2. If $A$ is a nonempty countable arithmetic set, then $A$ contains at least one arithmetic singleton.

Since every uncountable arithmetic set (in fact, every classical uncountable analytic set) contains a perfect subset, Corollary 2 is equivalent to Theorem 1. I do not know whether every member of a countable arithmetic set is an arithmetic singleton. This is also a problem presented by McLaughlin.

Proof of Theorem 1. We shall illustrate for the case that $A$ is a $\Pi_{5}^{0}$ set. Proof is analogous for the other cases. Note that if $A$ is a $\Sigma_{n+1}^{0}$ set then we can reduce it to the case of $\Pi_{n}^{0}$.

Received by the editors October 23, 1970.

AMS 1970 subject classifications. Primary 02F35, 02K30.

Key words and phrases. Arithmetic (i.e., $\Pi_{n}^{0}$ or $\Sigma_{n}^{0}$ ) subset of $N^{N}$, arithmetic singleton, dense-in-itself, perfect set.

(C) American Mathematical Society 1972 
Now let $A$ be a set defined by

$$
A=\left\{\alpha \in N^{N} \mid\left(\forall x_{0}\right)\left(\exists y_{0}\right)\left(\forall x_{1}\right)\left(\exists y_{1}\right) R\left(\alpha, x_{0}, x_{1}, y_{0}, y_{1}\right)\right\},
$$

where $R$ is $\Pi_{1}^{0}$. Then we have

$$
\begin{aligned}
\alpha \in A & \Leftrightarrow\left(\exists \beta_{0}\right)\left(\exists \beta_{1}\right)\left(\forall x_{0}\right)\left(\forall x_{1}\right) R\left(\alpha, x_{0}, x_{1}, \beta_{0}\left(x_{0}\right), \beta_{1}\left(x_{0}, x_{1}\right)\right) \\
& \Leftrightarrow(\exists \beta)(\forall x) R\left(\alpha,(x)_{0},(x)_{1}, \beta\left(\left\langle(x)_{0}\right\rangle\right), \beta\left(\left\langle(x)_{0},(x)_{1}, 1\right\rangle\right)\right),
\end{aligned}
$$

where $\left\langle a_{0}, a_{1}, \cdots, a_{k}\right\rangle=p_{0}^{a_{0}} \cdot p_{1}^{a_{1}} \cdots p_{k}^{a_{k}}$ and $p_{i}$ is the $(i+1)$ st prime number. (For notations used in this note, we mostly borrow from Kleene [3].) Let $R^{\prime}$ and $R^{\prime \prime}$ be predicates defined as follows:

$$
\begin{aligned}
R^{\prime}(\alpha, s) & \Leftrightarrow\left[\operatorname{Seq}(s) \wedge \operatorname{Lh}(s)=\left\langle(\operatorname{Lh}(s))_{0},(\operatorname{Lh}(s))_{1}, 2\right\rangle\right. \\
& \rightarrow R\left(\alpha,(\operatorname{Lh}(s))_{0},(\operatorname{Lh}(s))_{1}, \exp \left(s,\left\langle(\operatorname{Lh}(s))_{0}\right\rangle\right)-1,\right. \\
& \left.\quad \exp \left(s,\left\langle(\operatorname{Lh}(s))_{0},(\operatorname{Lh}(s))_{1}, 1\right\rangle\right)-1\right),
\end{aligned}
$$

where $\exp (s, i)=(s)_{i}$. And

where

$$
R^{\prime \prime}(\alpha, s) \Leftrightarrow(\forall i)_{i \leqq \operatorname{Lh}(s)} R^{\prime}(\alpha, \operatorname{rstr}(s, i)),
$$

$$
\begin{aligned}
\operatorname{rstr}(s, i) & =\prod_{k<i} p_{k}^{(s)_{k}}, & & \text { if } \operatorname{Seq}(s) \wedge i \leqq \operatorname{Lh}(s), \\
& =1, & & \text { otherwise. }
\end{aligned}
$$

Then $R^{\prime \prime}$ has the following properties:

(1) $\alpha \in A \Leftrightarrow(\exists \beta)(\forall x) R^{\prime \prime}(\alpha, \bar{\beta}(x))$,

(2) $R^{\prime \prime}$ is $\Pi_{1}^{0}$ and hence for each sequence number $s$, the set $E_{s}=$ $\left\{\alpha \mid R^{\prime \prime}(\alpha, s)\right\}$ is a closed set, and

(3) the Souslin system $\mathcal{S}=\left\{E_{s} \mid \operatorname{Seq}(s)\right\}$ is monotonic; that is, for all $\beta$ and $x, E_{\beta(x+1)} \subseteq E_{\beta(x)}$.

Now, as is usual with classical descriptive set theory, for a given sequence number $\bar{\gamma}(m)$, we shall define a set $A^{\bar{\gamma}(m)}$ as follows:

$$
\alpha \in A^{\bar{\gamma}(m)} \Leftrightarrow(\exists \beta)(\forall x) R^{\prime \prime}(\alpha, \bar{\gamma}(m) * \bar{\beta}(x)) .
$$

Then we have

$$
\begin{aligned}
& \alpha \in A^{\bar{\gamma}(m)} \Leftrightarrow(\exists \beta)(\forall x)(\forall i)_{i \leqq m+x} R^{\prime}(\alpha, \operatorname{rstr}(\bar{\gamma}(m) * \bar{\beta}(x), i)) \\
& \Leftrightarrow(\forall i)_{i \leqq m} R^{\prime}(\alpha, \bar{\gamma}(i)) \wedge(\exists \beta)(\forall i) R^{\prime}(\alpha, \bar{\gamma}(m) * \bar{\beta}(i)) \\
& \Leftrightarrow(\forall i)_{i \leqq m}\left[i=\left\langle(i)_{0},(i)_{1}, 2\right\rangle\right. \\
&\left.\rightarrow R\left(\alpha,(i)_{0},(i)_{1}, \gamma\left(\left\langle(i)_{0}\right\rangle\right), \gamma\left(\left\langle(i)_{0},(i)_{1}, 1\right\rangle\right)\right)\right] \\
& \wedge(\exists \beta)(\forall i)\left[m+i=\left\langle(m+i)_{0},(m+i)_{1}, 2\right\rangle\right. \\
& \rightarrow .\left\{\left\langle(m+i)_{0},(m+i)_{1}, 1\right\rangle<m\right. \\
& \rightarrow R\left(\alpha,(m+i)_{0},(m+i)_{1}, \gamma\left(\left\langle(m+i)_{0}\right\rangle\right),\right. \\
&\left.\left.\gamma\left(\left\langle(m+i)_{0},(m+i)_{1}, 1\right\rangle\right)\right)\right\}
\end{aligned}
$$




$$
\begin{gathered}
\dot{\wedge}\left\{\left\langle(m+i)_{0}\right\rangle<m \wedge\left\langle(m+i)_{0},(m+i)_{1}, 1\right\rangle \geqq m\right. \\
\rightarrow R\left(\alpha,(m+i)_{0},(m+i)_{1}, \gamma\left(\left\langle(m+i)_{0}\right\rangle\right),\right. \\
\left.\left.\beta\left(\left\langle(m+i)_{0},(m+i)_{1}, 1\right\rangle-m\right)\right)\right\} \\
\wedge\left\{\left\langle(m+i)_{0}\right\rangle \geqq m\right. \\
\rightarrow R\left(\alpha,(m+i)_{0},(m+i)_{1}, \beta\left(\left\langle(m+i)_{0}\right\rangle-m\right),\right. \\
\left.\left.\left.\beta\left(\left\langle(m+i)_{0},(m+i)_{1}, 1\right\rangle-m\right)\right)\right\}\right] .
\end{gathered}
$$

The second member of the outermost conjunction in the latter formula is equivalent to

$$
\begin{aligned}
\left(\exists \beta_{0}\right)\left(\exists \beta_{1}\right)\left(\forall x_{0}\right)\left(\forall x_{1}\right)[ & \left\{\left\langle x_{0}, x_{1}, 1\right\rangle<m \rightarrow R\left(\alpha, x_{0}, x_{1}, \gamma\left(\left\langle x_{0}\right\rangle\right), \gamma\left(\left\langle x_{0}, x_{1}, 1\right\rangle\right)\right)\right\} \\
\wedge & \left\{\left\langle x_{0}\right\rangle<m \wedge\left\langle x_{0}, x_{1}, 1\right\rangle \geqq m\right. \\
& \left.\rightarrow R\left(\alpha, x_{0}, x_{1}, \gamma\left(\left\langle x_{0}\right\rangle\right), \beta_{1}\left(\left\langle x_{0}, x_{1}, 1\right\rangle-m\right)\right)\right\} \\
\wedge & \left\{\left\langle x_{0}\right\rangle \geqq m\right. \\
& \left.\left.\rightarrow R\left(\alpha, x_{0}, x_{1}, \beta_{0},\left(\left\langle x_{0}\right\rangle-m\right), \beta_{1}\left(\left\langle x_{0}, x_{1}, 1\right\rangle-m\right)\right)\right\}\right] \\
\Leftrightarrow\left(\forall x_{0}\right)\left(\exists y_{0}\right)\left(\forall x_{1}\right)\left(\exists y_{1}\right)[ & \left\{\left\langle x_{0}, x_{1}, 1\right\rangle<m\right. \\
\rightarrow & \left.R\left(\alpha, x_{0}, x_{1}, \gamma\left(\left\langle x_{0}\right\rangle\right), \gamma\left(\left\langle x_{0}, x_{1}, 1\right\rangle\right)\right)\right\} \\
\wedge\left\{\left\langle x_{0}\right\rangle\right. & \left.<m \wedge\left\langle x_{0}, x_{1}, 1\right\rangle \geqq m \rightarrow R\left(\alpha, x_{0}, x_{1}, \gamma\left(\left\langle x_{0}\right\rangle\right), y_{1}\right)\right\} \\
\wedge & \left.\wedge\left\{\left\langle x_{0}\right\rangle \geqq m \rightarrow R\left(\alpha, x_{0}, x_{1}, y_{0}, y_{1}\right)\right\}\right] .
\end{aligned}
$$

(Note that $\bar{\gamma}(m)$ is a given fixed sequence number.) Therefore, for each sequence number $s, A^{s}$ is an arithmetic subset of $N^{N}$, too. Further, by the definition (4) we have

$$
A^{\left[a_{0}, a_{1}, \cdots, a_{k}\right]}=\bigcup_{n=0}^{\infty} A^{\left[a_{0}, a_{1}, \cdots, a_{k}, n\right]},
$$

where we denote $\left\langle a_{0}+1, a_{1}+1, \cdots, a_{k}+1\right\rangle$ by $\left[a_{0}, a_{1}, \cdots, a_{k}\right]$.

Now suppose that no member of $A$ constitutes an arithmetic singleton. Let $\alpha \in A$. Since $A=\bigcup_{n=0}^{\infty} A^{[n]}$, there is an $n_{0}$ such that $\alpha \in A^{\left[n_{0}\right]} . A^{\left[n_{0}\right]}$ does not contain any arithmetic singleton, since its overset $A$ does not. As seen above, $A^{\left[n_{0}\right]}$ is also an arithmetic set and hence it contains no isolated elements. Therefore $A^{\left[n_{0}\right]}$ is dense-in-itself. So, for each number $m_{0}$, $A^{\left[n_{0}\right]} \cap \delta\left(\bar{\alpha}\left(\left[m_{0}\right]\right)\right)$ is nonempty and dense-in-itself, where $\delta(s)$ denotes the Baire interval determined by a sequence number $s$. Let us put

$$
B^{\left[m_{0}\right]}=A^{\left[n_{0}\right]} \text { and } F_{\left[m_{0}\right]}=E_{\left[n_{0}\right]}
$$

for all $m_{0}$. From each set $B^{\left[m_{0}\right]} \cap \delta\left(\bar{\alpha}\left(\left[m_{0}\right]\right)\right)$ we can choose an element $\alpha_{\left[m_{0}\right]}$ such that the $\alpha_{\left[m_{0}\right]}$ 's satisfy the following conditions:

$$
\alpha_{\left[m_{0}\right]} \neq \alpha, \quad \alpha_{\left[m_{0}\right]} \neq \alpha_{\left[m_{0}^{\prime}\right]} \text { if } m_{0} \neq m_{0}^{\prime} .
$$


Since $B^{\left[m_{0}\right]}=\bigcup_{n=0}^{\infty} A^{\left[n_{0}, n\right]}$, for each $m_{0}$ there is an $n_{1}$ such that $\alpha_{\left[m_{0}\right]} \in$ $A^{\left[n_{0}, n_{1}\right]}$. Let us put

$$
B^{\left[m_{0}, m_{1}\right]}=A^{\left[n_{0}, n_{1}\right]} \text { and } F_{\left[m_{0}, m_{1}\right]}=E_{\left[n_{0}, n_{1}\right]}
$$

for all $m_{1}$. Then $B^{\left[m_{0}, m_{1}\right]} \cap \delta\left(\bar{\alpha}_{\left[m_{0}\right]}\left(\left[m_{0}+m_{1}+1\right]\right)\right)$ is nonempty and densein-itself. From each set $B^{\left[m_{0}, m_{1}\right]} \cap \delta\left(\bar{\alpha}_{\left[m_{0}\right]}\left(\left[m_{0}+m_{1}+1\right]\right)\right)$, we can choose an $\alpha_{\left[m_{0}, m_{1}\right]}$ such that $\alpha_{\left[m_{0}, m_{1}\right]}$ 's satisfy the following conditions:

$$
\begin{aligned}
& \alpha_{\left[m_{0}, m_{1}\right]} \neq \alpha ; \quad \alpha_{\left[m_{0}, m_{1}\right]} \neq \alpha_{\left[m_{0}^{\prime}\right]} ; \\
& \alpha_{\left[m_{0}, m_{1}\right]} \neq \alpha_{\left[m_{0}^{\prime}, m_{1}^{\prime}\right]} \quad \text { if }\left[m_{0}, m_{1}\right] \neq\left[m_{0}^{\prime}, m_{1}^{\prime}\right] .
\end{aligned}
$$

And so on. Thus we obtain elements $\alpha_{\left[m_{0}, m_{1}, \cdots, m_{k}\right]} \in A$ for $k, m_{i}=0,1$, $2, \cdots$, and they possess the following properties:

$$
\begin{gathered}
\alpha_{\left[m_{0}, \cdots, m_{k}\right]} \neq \alpha_{\left[m_{0}^{\prime}, \cdots, m_{j}^{\prime}\right]} \text { if }\left[m_{0}, \cdots, m_{k}\right] \neq\left[m_{0}^{\prime}, \cdots, m_{j}^{\prime}\right], \\
\alpha_{\left[m_{0}, \cdots, m_{k}\right]} \in B^{\left[m_{0}, \cdots, m_{k}\right]}=A^{\left[n_{0}, \cdots, n_{k}\right]} \subseteq E_{\left[n_{0}, \cdots, n_{k}\right]}=F_{\left[m_{0}, \cdots, m_{k}\right]},
\end{gathered}
$$

where $\left[n_{0}, \cdots, n_{k}\right]$ is determined by $\left[m_{0}, \cdots, m_{k}\right]$,

$$
\alpha_{\left[m_{0}, \cdots, m_{k}, m_{k+1}\right]} \in \delta\left(\bar{\alpha}_{\left[m_{0}, \cdots, m_{k}\right]}\left(\left[m_{0}+\cdots+m_{k+1}+k+1\right]\right)\right) .
$$

Let $Q=\left\{\alpha_{s} \mid \operatorname{Seq}(s)\right.$ and $\left.\operatorname{Lh}(s)>0\right\}$. Then $Q$ is dense-in-itself and hence its derived set $Q^{\prime}$ is a perfect set. Using (1)-(3) and (6)-(8) we can show that $Q^{\prime}$ is contained in $A$. In proving this fact, note that each $E_{s}$ is a closed set. (For details, see Hahn [1, pp. 356-358].) Therefore $A$ contains a perfect subset. This completes the proof of Theorem 1 .

Since the final expression for $\alpha \in A^{\bar{\gamma}^{(m)}}$ in the preceding proof is also $\Pi_{5}^{0}$, we have shown that if $A$ is a nonempty $\Pi_{5}^{0}$ set with no $\Pi_{5}^{0}$ singleton then $A$ contains a perfect subset. ${ }^{1}$ Thus we obtain the following theorem:

THEOREM 3. Every nonempty countable $\Sigma_{n+1}^{0}$ set contains a $\Pi_{n}^{0}$ singleton.

\section{REFERENCES}

1. H. Hahn, Reelle Funktionen, Akademie Verlagsgesellschaft, Leipzig, 1932; reprint, Chelsea, New York, 1948.

2. J. Harrison, Recursive pseudo-well-orderings, Trans. Amer. Math. Soc. 131 (1968), 526-543. MR 39 \#5366.

3. S. C. Kleene, Arithmetical predicates and function quantifiers, Trans. Amer. Math. Soc. 79 (1955), 312-340. MR 17, 4.

4. A. R. D. Mathias, A survey of recent results in set theory, Proc. Sympos. Pure Math., vol. 13, part 2 (to appear).

College of Engineering, Hosei University, Tokyo, Japan

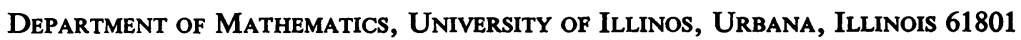

${ }^{1}$ This is based on a suggestion of Professor McLaughlin. 